\title{
Can immune parameters be used as predictors to distinguish between pulmonary multidrug-resistant and drug-sensitive tuberculosis?
}

\author{
Bayram Kiran ${ }^{1}$, Tulin Cagatay ${ }^{2}$, Philip Clark ${ }^{3}$, Filiz Kosar ${ }^{4}$, Penbe Cagatay ${ }^{5}$, Sibel Yurt ${ }^{4}$, \\ Faruk Suzergoz ${ }^{6}$, Ali Osman Gurol ${ }^{7}$
}

\author{
1Department of Microbiology, Virology and Basic Immunology Division, \\ Istanbul Medical Faculty, Istanbul University, Istanbul, Turkey \\ 2Department of Pulmonary Diseases, Istanbul Medical Faculty, Istanbul University, \\ Istanbul, Turkey \\ ${ }^{3}$ Faculty of Pharmacy, Yeditepe University, Istanbul, Turkey \\ ${ }^{4}$ Yedikule Chest Diseases and Chest Surgery Education and Research Hospital, \\ Istanbul, Turkey \\ ${ }^{5}$ Cerrahpasa Medical Faculty, Department of Biostatistics, Istanbul University, \\ Istanbul, Turkey \\ ${ }^{6}$ Division of Biology, Science-Art Faculty, Harran University, Sanliurfa, Turkey \\ 7Department of Immunology, Institute of Experimental Medicine, Istanbul University, \\ Istanbul, Turkey
}

Submitted: 24 February 2009

Accepted: 1 July 2009

Arch Med Sci 2010; 6, 1: 77-82

DOI 10.5114/aoms.2010.13511

Copyright (c) 2010 Termedia \& Banach

\section{Abstract}

Introduction: Despite the development and wide implementation of Directly Observed Therapy Strategies (DOTS), multidrug-resistant tuberculosis (MDR-TB) remains a serious global health threat. In this study, the role of host immune response in patients with MDR-TB is investigated and compared with that of patients with smear-positive drug-sensitive tuberculosis (SP-TB).

Material and methods: 27 patients with SP-TB, 20 patients with MDR-TB, and 20 healthy controls were included in the study. Immune parameters were determined by flow cytometry using monoclonal antibodies in order to compare the percentage values of these markers in the two study groups and the control group.

Results: The levels of lymphocyte subgroups in the gate of CD45(+)/CD14(-) lymphocyte: CD45(+), CD3(+), CD4(+), NK, CD3/HLA-DR, CD 95(+) cells were significantly lower; by contrast CD23(+), CD25(+), CD19(+), CD4(+)/CD8(+), HLA-DR cells were found to be lower, but not significantly so in patients with MDR-TB, compared to levels in patients in the SP-TB and control groups. Besides these findings, the levels of NKT cells and $\gamma \delta \mathrm{TCR}(+)$ cells were significantly higher in the MDR-TB than in the healthy control and SP-TB group.

Conclusions: The lower levels of CD3/HLA-DR, CD4 (+), Fas (+), and NK, and the higher level of NKT together with $\gamma \delta$ T cells in patients with MDR-TB compared to those in SP-TB may indicate a profound immune suppression in MDR-TB patients and thereby may denote an accumulation in the bacterial load. Our findings may shed light on the pathogenesis and prognosis of MDR tuberculosis, and may point towards the use of flow cytometry findings as an aid to early diagnosis in MDR-TB patients.

Key words: HLA-DR, $\gamma \delta$ T cell, NK-T cell, multidrug-resistant tuberculosis.

\author{
Corresponding author: \\ Dr. Bayram Kiran \\ Department of Microbiology \\ Virology and Basic \\ Immunology Division \\ Istanbul Medical Faculty \\ Istanbul University \\ Capa, Istanbul, Turkey \\ Phone: +90 2124142000 \\ (ext. 32144) \\ E-mail: \\ drbayramkiran@yahoo.com
}




\section{Introduction}

Despite the development of the directly observed therapy strategy (DOTS) multidrug-resistant tuberculosis (MDR-TB) is still an important problem that threatens global community health [1-3]. Nearly 50 billion people are infected with MDR-TB worldwide and the number is increasing every year as new cases are infected with MDR-TB strains [4].

Most of the risk factors for the emergence of drug resistance in tuberculosis are acquired. For example, inadequate treatment due to adverse drug reactions, economic hardships, and non-adherence to drug therapy when symptoms resolve may be responsible for the current high prevalence of MDR-TB.

In addition, there is a lack of clarity regarding the genetic control of tuberculosis which, if resolved, could help to explain why active tuberculosis disease only emerges in a relatively small number of infected people [5].

Protection against Mycobacterium tuberculosis $(M . t b c)$ is based on cell-mediated immunity, most importantly involving $\mathrm{CD}_{4}(+)$ and $\mathrm{CD}_{8}(+)$ T-cell subsets [6]. The CD4 $T$ cell is believed to be responsible for regulating the immune response to $M$. tbc. When activated, CD4 cells proliferate and produce cytokines which in turn activate mononuclear phagocytes to control the growth of intracellular mycobacteria [7].

The primary function of the CD8 (+) lymphocytes (T suppressor/cytotoxic) is the lysis and apoptosis of the infected cells. In some studies, it was found that in active tuberculosis patients, $C_{4}$ and $C D_{8}$ counts are decreased, but the $\mathrm{CD}_{4} / \mathrm{CD}_{8}$ ratio is increased compared to the healthy population [8].

Findings in peripheral blood contrast with other research suggesting an increase in CD4 cells and a reduction in CD8 cells in bronchoalveolar lavage fluid leading to an increased CD4/CD8 ratio, particularly where there is moderate or advanced pulmonary involvement. The researchers attributed these findings to compartmentalization or sequestration of CD4+ lymphocytes in cases of high grade pulmonary TB [9].

The human leukocyte antigen (HLA) system is a powerful genetic marker. Host immune response and infectious diseases are genetically controlled by the HLA system [10].

In M. tbc infection, HLA factors are associated with the type and severity of the disease. Although immune suppression and the progress of disease are controlled by the HLA system, innate resistance to Mycobacterium infection appears to be dependent on non-HLA genes [11, 12]. However, genetic susceptibility of the host to multidrug-resistant tuberculosis (MDR-TB) is not fully understood [13].

In this study, we aimed to compare the levels of CD3+/HLA-DR, HLA-DR and lymphocyte subgroups
(CD45, CD3, CD4, CD19, CD 23, $\gamma \delta$-T cell, NK-T) and the marker of apoptosis Fas (CD95) in smearpositive drug-sensitive tuberculosis (SP-TB) with these values in smear-positive MDR-TB cases.

Where the immune profiles of the two groups were different, the effect of immune status in the development and clinical progress of MDR-TB was examined.

\section{Material and methods}

\section{Patients and study design}

20 healthy controls, 27 patients with sputum smear-positive pulmonary tuberculosis sensitive to primary anti-TB therapy, and 20 patients with sputum smear-positive MDR-TB were included in the study.

All of the cases were HIV (-). In the MDR-TB group, two of the cases had not been previously treated with anti-tuberculosis agents (primary MDR), while 18 of the cases had been treated at least two times before the study (secondary MDR).

Where possible, patients without any comorbidities were selected. In all cases, baseline data such as history (both medical and family histories, including TB family history), physical examination, biochemical parameters and complete blood count (particularly haemoglobin [Hb], haematocrit [Htc] and leukocyte values) were recorded.

Mycobacterium tuberculosis was isolated from the sputum and cultured according to the standard techniques, and sensitivity testing was performed using the absolute concentration method [14, 15].

\section{Immune profile determination}

The patients' immune profiles were determined by analyzing blood samples, by whole blood lysis on flow cytometry (Becton-Dickinson FACSCalibur, San Jose, CA) following heparinization and treatment with fluorochrome-conjugated monoclonal antibodies (anti-human IgG1-FITC/IgG2a PE isotype control, anti-human CD45-FITC, CD3-FITC, CD4-FITC, CD8-PE, CD19-PE, CD16/56-PE(NK), HLA-DR, CD23-PE, CD25-FITC, Fas(CD95)-PE and $\gamma \delta$-T cell-PE). In order to detect the changes in percentage values of these markers, first $100 \mu$ l of heparinized blood was placed into all test tubes, and then $10 \mu \mathrm{l}$ of isotype control was added into one tube and $10 \mu \mathrm{l}$ of monoclonal antibody into the others. After incubating for $30 \mathrm{~min}$ at room temperature in a dark room, erythrocytes were removed using $500 \mu \mathrm{l}$ of erythrocyte lysis buffer and $5 \mathrm{ml}$ of phosphate buffer saline (PBS) to wash the solution twice before centrifuging for $5 \mathrm{~min}$ at $1850 \mathrm{rpm}$. Then a sample containing 10,000 cells from each tube was counted in the appropriate lymphocyte gate using flow cytometry equipped with 
a CELLQuest software program. Finally, the percentage value of all the markers was determined using the analysis program.

\section{Statistical analysis}

Statistical analysis was conducted using Unistat 5.1 software. All numerical values are reported as means \pm SD. A comparison of variables between three groups was performed using one-way ANOVA. Differences between control, SP-TBC and MDR-TB groups were determined by the Bonferroni test. Chest $X$-ray, family and TB history groups were estimated by $\chi^{2}$ test. $P$-values less than 0.05 were considered significant.

\section{Results}

\section{Patients' demographic details}

As can be seen in Table I, leucocytes counts were found to be significantly different between groups and increases were observed in both SP-TB and MDR-TB groups. Haemoglobin and haematocrit levels were found to be increased in the SP-TB group and decreased in the MDR-TB group, compared to the control group.

Most of the MDR-TB patients had a positive family TB history, while less distinct family histories of tuberculosis were noted in SP-TB and control groups, as shown in Table I.
Bilateral lesions were found to be dominant in the MDR-TB group, while unilateral lesions were dominant in the SP-TB group. ARB and bacillus cultures were found in all of the patients in SP-TB and MDR-TB groups, while none of the control individuals had positive findings for ARB or bacillus cultures, as can also be observed from Table I.

\section{Flow cytometric analysis}

CD4, CD3, Fas (CD95), CD3/CD25 and CD3/ HLD-DR expressions were found to be significantly decreased in both SP-TB and MDR TB groups compared to the control group (Figures 1, 2). In contrast to these findings, NKT (CD3 (+) and $\gamma \delta \mathrm{T}$ cell levels were found to be significantly increased, while CD8 levels were non-significantly increased, in both SP-TB and MDR-TB groups compared to the control group, as shown in Figures 1 and 2.

CD45, CD19, CD16/56 (NK), HLA-DR, CD4/CD8 and $C D 3 / C D 23$ levels were found to be increased in the SP-TB group and significantly decreased in the MDR-TB group compared to the control group (Figures 1, 2).

\section{Discussion}

The latest $\mathrm{WHO}$ estimates indicate that between 250,000 and 500,000 new MDR-TB cases occur annually, which accounts for $3-6 \%$ of all new and

Table I. Demographic, haematological, radiological and microbiological characteristics of control individuals, SP-TB and MDR-TB patients

\begin{tabular}{|c|c|c|c|c|c|}
\hline & & $\begin{array}{l}\text { Control } \\
(n=20)\end{array}$ & $\begin{array}{l}\text { SP-TB } \\
(n=27)\end{array}$ & $\begin{array}{l}\text { MDR-TB } \\
(n=20)\end{array}$ & Sig. \\
\hline Age & & $44.1 \pm 7.0$ & $35.9 \pm 13.9$ & $40.6 \pm 12.6$ & $0.08^{a}$ \\
\hline Sex & $(M / F)$ & 7/13 & $24 / 3$ & $17 / 3$ & $0.001^{b}$ \\
\hline Smoking & (p/year) & $7.3 \pm 1.3$ & $19.1 \pm 3.9$ & $20.0 \pm 3.3$ & $0.06^{a}$ \\
\hline Leukocyte & $\left(\mathrm{mm}^{3}\right)$ & $6961.8 \pm 331.9$ & $9292.5 \pm 460.2^{c}$ & $9575.0 \pm 525.1^{d}$ & $0.001^{a}$ \\
\hline Haemoglobin & (gr/dl) & $12.0 \pm 0.2$ & $12.9 \pm 0.2$ & $11.47 \pm 0.30^{e}$ & $0.001^{a}$ \\
\hline Haematocrit & (\%) & $38.3 \pm 0.5$ & $39.4 \pm 0.8$ & $36.0 \pm 0.7 \mathrm{e}$ & $0.009^{b}$ \\
\hline \multicolumn{6}{|l|}{ Family TB history } \\
\hline \multirow[t]{2}{*}{ TB negative } & $(n)$ & 16 & 20 & 5 & \multirow{4}{*}{$0.001^{b}$} \\
\hline & (\%) & 84.2 & 74.1 & 29.4 & \\
\hline \multirow[t]{2}{*}{ TB positive } & $(n)$ & 3 & 7 & 12 & \\
\hline & (\%) & 15.8 & 25.9 & 70.6 & \\
\hline \multicolumn{6}{|l|}{ Chest $X$ ray } \\
\hline \multirow[t]{2}{*}{ Unilateral lesion } & $(n)$ & - & 17 & 9 & \multirow{4}{*}{$0.001^{b}$} \\
\hline & (\%) & - & 65.4 & 34.6 & \\
\hline \multirow[t]{2}{*}{ Bilateral lesion } & (n) & - & 4 & 16 & \\
\hline & (\%) & - & 20 & 80 & \\
\hline ARB & $(n)$ & - & 27 & 20 & \multirow{2}{*}{$0.0001 b$} \\
\hline & (\%) & - & 100 & 100 & \\
\hline \multirow[t]{2}{*}{ Bacillus culture positive } & $(n)$ & - & 27 & 20 & \multirow[t]{2}{*}{$0.0001 b$} \\
\hline & (\%) & - & 100 & 100 & \\
\hline
\end{tabular}

Values are represented as mean $\pm S D$. Comparisons performed by aANOVA, $p$ values calculated by ${ }^{b} \chi^{2}$ test, $c p<0.05$ in comparison to control group, ${ }^{d} p<0.05$ in comparison to SP-TB group, ${ }^{e} p<0.05$ significance between SP-TB and MDR-TB groups, comparisons performed by cdeBonferroni test

SP-TB - drug-sensitive tuberculosis, MDR-TB - multidrug-resistant tuberculosis, ARB - acido-resistant bacilli 


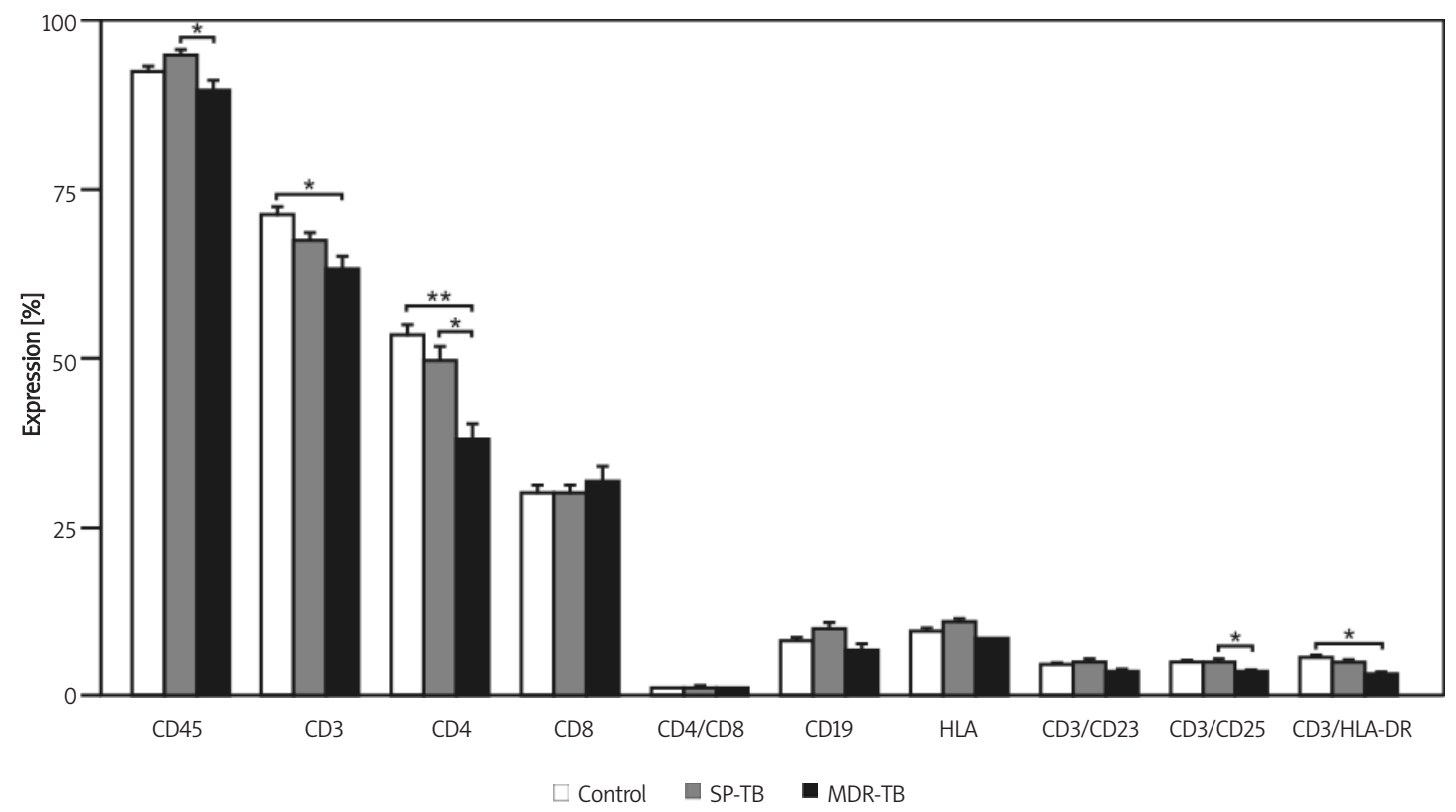

Figure 1. T and B cell surface antigen expression on lymphocytes from control individuals, SP and MDR-TBC patients ${ }^{*} p<0.05,{ }^{* *} p<0.001$, significances determined by Bonferroni test, SP-TB - drug-sensitive tuberculosis, MDR-TB - multidrug-resistant tuberculosis

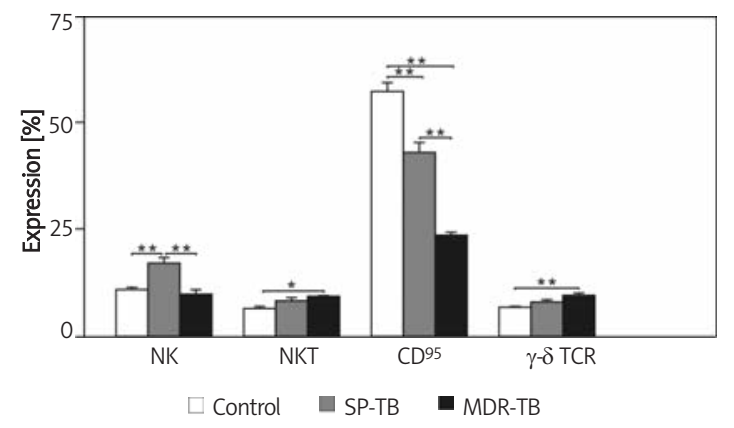

Figure 2. Killer cell subsets, Fas and $\gamma \delta$ TCR expression in control individuals, SP and MDR-TBC patients

${ }^{*} p<0.05,{ }^{* *} p<0.001$, significances determined by Bonferroni test, SP-TB - drug-sensitive tuberculosis, MDR-TB - multidrug-resistant tuberculosis

previously treated TB cases. These figures indicate an upturn in the proportion of resistant isolates and the authors of the review also confirm the emergence of extensively resistant TB which includes resistance to fluoroquinolones and aminoglycosides [16]. The emergence and spread of drug resistance is widely recognized as posing a public health threat of global proportions. There is a clear correlation between late diagnosis of disease which causes a delay in initiating effective treatment, and unsuccessful clinical outcomes [17]. For this reason, immunological testing may have an important role in enabling prompt diagnosis and treatment of tuberculosis.

The total blood leukocyte counts and total lymphocyte (CD45) levels of patients varied between all the groups; and particularly when the MDR-TB and the SP-TB group were compared the difference was statistically significant. Leukocyte levels were higher in patient groups compared to controls, while CD45 counts were lowest in the MDR-TB group. This level represents a small proportion of the total leukocyte numbers in patients with MDR-TB, thus indicating the presence of lymphopenia. This low CD45 level in the MDRTB group draws attention to these patients' reduced lymphocyte response, compared to that of the SP-TB group. In addition, the lymphopenia observed among patients with MDR-TB may play an important role in the pathogenesis of the disease.

The lowest levels of both HLA-DR and CD3/ HLA-DR were seen in the MDR-TB group. These measurements reflect the low level of active lymphocytes in patients with MDR-TB. Our findings are also consistent with a study by Rodriguez et al., which did not find any statistically significant difference in the HLA-DR (+) expression index in PPD (-), PPD (+), active tuberculosis, or treated tuberculosis groups [8].

In patients with MDR-TB, CD4 (+) T lymphocyte counts have been observed to be low. On the other hand, some studies indicate that CD3 (+) and CD4 (+) cell counts may be higher in drug-sensitive pulmonary TB cases $[18,19]$.

T lymphocytes, especially CD4 (+) T lymphocytes, play an important role in the immune reaction against MDR-TB. It is not clearly known whether the effects of CD4 (+) lymphocytes to control disease are mediated by their cytokine secretions or their 
direct effect on macrophages. Indeed there are many inter- and intracellular processes involved; and if some of these mechanisms can be elucidated, new treatment strategies may be developed.

One of the aims of our study was to find out whether we could predict the development of MDR-TB by looking at the HLA-DR and CD3/ HLA-DR levels. When the three groups were compared, although the differences between the HLA-DR levels were not significant, a significant difference was found in CD3/HLA-DR levels for patients with MDR-TB.

CD8 (+) T cells are essential for host defence against intracellular bacterial pathogens such as M. tub. $[18,20]$. Yildiz et al. found significantly lower values of lymphocyte numbers, absolute numbers and percentages of CD3 (+) and CD4 (+) in MDR-TB cases when compared to SP-TB cases. Besides this, the CD4/CD8 ratio was lower in MDR-TB cases, when compared with SP-TB, but these results were not statistically significant [21]. In our study, while not statistically significant, the high levels of cytotoxic CD8 (+) lymphocytes in patients with MDR-TB may be secondary to the stimulation of tuberculoid antigens. Alternatively an increase in suppressor CD8 lymphocytes may be the causative factor. This increase in CD8+ supports the suppression reflected in the reduction of the CD4/CD8 ratio from 1.44 in the control group, and 1.36 in the SP-TB group to 1.08 in the MDR-TB group.

Levels of the CD19 (+) B lymphocytes responsible for antibody production and their activation receptor CD23 were non-significantly reduced in the MDR-TB group. This suggests that humoral as well as cellular immunity is affected, and that this effect is more profound in the MDR-TB group than the SP-TB group. Similarly, Cagatay et al. showed that both humoral and cellular immune response is suppressed in patients with MDR-TB [22].

Fas (CD95)(+) lymphocytes were found to be significantly lower in MDR-TB patients compared to the control group and the SP-TB patients. Fas (CD95)(+) lymphocyte is typical for patients with prognostically unfavourable immune abnormalities. It is tempting to speculate that apoptosis of T cells (possibly Th1 cells) is of great importance in the pathogenesis of the immunodeficiency associated with the mycobacteriosis [23]. In our study, Fas (CD95)(+) among the three groups was highly significant, with patients with MDR-TB demonstrating the lowest level. This may indicate suppression of the host immune response and may help to shed light on MDR-TB pathogenesis.

In our study, NK (CD16/56) cells were increased in the SP-TB group compared to healthy controls. On the other hand, when compared to both controls and the SP-TB group, the MDR-TB group demonstrated a profound reduction in NK levels, indicating a dysfunctional NK response. Yildiz et al. demonstrated that patients with MDR-TB had significantly lower NK compared to normal TB and controls. This reduction in NK may suggest a role for impaired NK function in the pathogenesis of MDR-TB [22, 23].

The level of human natural killer T cells (NKT) is a non-invasive marker of pulmonary TB activation and disease prognosis. The reason for a higher level of NKT in tuberculosis patients might be related to the high load of phosphorylated metabolites released following the phagocytosis of bacilli by macrophages [24-26].

In this study, both groups of patients with TB had significantly raised NKT levels, indicating an active infection and a host immune protective response against mycobacteria compared to healthy controls. Thus, the NKT cell increase is consistent with the presence of active infection as seen in our patients. In addition, NKT levels may shed light on disease progression and prognosis, and help to distinguish MDR-TB from SP-TB disease. The mucosal immune system showed a similarly significant increase in both SP-TB cases and in patients with MDR-TB. Lockhart et al. and Lee et al. demonstrated that in infection with Mycobacterium, $\gamma \delta$ T cells become dominant as they are activated by phosphorylated metabolites within phagosomes $[27,28]$. In our study, there was a significant increase in the level of $\gamma \delta$ T cells in the MDR-TB group compared to the control and SP-TB groups.

To summarize these flow cytometric findings, MDR M. tub. modifies host defence by suppressing CD3/HLA-DR+ and Fas + T cells, but by increasing $\gamma \delta$-T cells and NKT cells in MDR-TB patients.

These findings may shed light on MDR-TB pathogenesis, although it cannot be elucidated from the findings whether the immune status of the patients is reflective of their condition before or after they succumbed to active MDR-TB infection. However, all of the immune parameters seem to point to a worse prognosis in patients with MDR-TB compared to drug-sensitive tuberculosis. It may also be possible to gain valuable information by measuring the immune parameters of individuals who had contacted patients with a family history of MDR-TB.

In conclusion, decreased levels of CD4, CD3/ HLA-DR+ and Fas + T cells and increased levels of NKT and $\gamma \delta$ T cells detected by flow cytometry in MDR-TB cases compared to drug-sensitive TB patients might have diagnostic value. Based on immunological profiles it may be assumed that the patient has developed MDR-TB, and so without the two-month delay of culture and sensitivity testing, empirical therapy may be started. This proposition of course remains to be confirmed by further research and tested in clinical practice. 


\section{References}

1. Blöndal K. Barriers to reaching the targets for tuberculosis control: multidrug-resistant tuberculosis. Bull World Health Organ 2007; 85: 387-90.

2. Uffredi ML, Truffot-Pernot C, Dautzenberg B, Renard M, Jarlier V, Robert J. An intervention programme for the management of multidrug-resistant tuberculosis in France. Int J Antimicrob Agents 2007; 29: 434-9.

3. Chaudhury RR, Thatte U. Beyond DOTS: avenues ahead in the management of tuberculosis. Natl Med J India 2003; 16: 321-7.

4. Katalinić-Janković V, Obrovac M. Drug-resistant tuberculosis: resistance mechanisms and drug susceptibility of Mycobacterium tuberculosis. Acta Med Croatica 2004; 58: 323-8.

5. Hu DY, Wang T, Liu XY, et al. Study on the risk factors leading to irregularly anti-pulmonary tuberculosis drug taking in patients with smear positive [Chinese]. Zhonghua Liu Xing Bing Xue Za Zhi 2007; 28: 237-40.

6. Deveci F, Akbulut $\mathrm{HH}$, Celik I, Muz MH, Ilhan F. Lymphocyte subpopulation in pulmonary tuberculosis patients. Mediators Inflamm 2006; 2006: 89070.

7. Orme IM, Andersen P, Boom WH. T cell response to Mycobacterium tuberculosis. J Infect Dis 1993; 167: 1481-97.

8. Rodrigues DS, Medeiros EA, Weckx LY, Bonnez W, Salomao R, Kallas EG. Immunophenotypic characterization of peripheral $T$ lymphocytes in Mycobacterium tuberculosis infection and disease. Clin Exp Immunol 2002; 128: 149-54

9. Tsao TC, Chen CH, Hong JH, Hsieh MJ, Tsao KC, Lee CH. Shifsts of T4/T8 lymphocytes from BAL fluid and peripheral blood by clinical grade in patients with pulmonary tuberculosis. Chest 2002; 122: 1285-91.

10. Hwang CH, Khan S, Ende N, Mangura BT, Reichman LB, Chou J. The HLA-A, -B, and -DR phenotypes and tuberculosis. Am Rev Respir Dis 1985; 132: 382-5.

11. Bothamley GH, Beck JS, Schreuder GM, et al. Association of Tuberculosis and M. Tuberculosis-specific antibody levels with HLA. J Infect Dis 1989; 159: 549-55.

12. Meyer CG, May J, Stark K. Human leukocyte antigens in tuberculosis and leprosy. Trends Microbiol 1998; 6: 148-54.

13. Sharma SK, Turaga KK, Balamurugan A, et al. Clinical and genetic risk factors for the development of multi-drug resistant tuberculosis in non-HIV infected patients at a tertiary care center in India: a case-control study. Infect Genet Evol 2003; 3: 183-8.

14. Drobniewski F, Rüsch-Gerdes S, Hoffner S. Antimicrobial susceptibility testing of Mycobacterium tuberculosis Report of the Subcommittee on Antimicrobial Susceptibility Testing of Mycobacterium tuberculosis of the European Committee for Antimicrobial Susceptibility Testing (EUCAST) of the European Society of Clinical Microbiology and Infectious Diseases (ESCMID). Clin Microbiol Infect 2007; 13: 1144-56.

15. Newman MJ, Addo KK, Aboagye S, et al. Culture and sensitivity of mycobacterial isolates from cases of pulmonary tuberculosis classified as treatment failures in a teaching hospital. West Afr J Med 2007; 26: 131-3.

16. Chan ED, Iseman MD. Multidrug-resistant and extensively drug-resistant tuberculosis: a review. Curr Opin Infect Dis 2008; 21: 587-95.

17. Mitnick C, Bayona J, Palacios E, et al. Community-based therapy for multidrug-resistant tuberculosis in Lima, Peru. Engl J Med 2003; 348: 119-28.
18. Yoneda T, Ellner JJ. CD4(+) T cell and natural killer celldependent killing of Mycobacterium tuberculosis by human monocytes. Am J Respir Crit Care Med 1998; 158: 395-403.

19. Montes Santiago J, Gambon DF, Pacheco CM, Cerda MT. Cellular immune response in tuberculosis: analysis of $T$ lymphocytes and their subsets, B lymphocytes and natural cytotoxic cells in different tuberculosis states and body fluids. Rev Clin Esp 1996; 196: 223-7.

20. Lewinsohn DA, Winata E, Swarbrick GM, et al. Immunodominant tuberculosis CD8 antigens preferentially restricted by HLA-B. PLoS Pathog 2007; 3: 1240-9.

21. Yildiz P, Kadakal F, Tutuncu Y, et al. Natural killer cell activity in multidrug-resistant pulmonary tuberculosis. Respiration 2001; 68: 590-4.

22. Cagatay T, Kiran B, Yurt S, Gulbaran Z, Kosar F, Cagatay P. Levels of tumour necrosis factor-alpha and IL-1alpha in newly diagnosed and multi-drug resistant tuberculosis. Respirology 2005; 10: 290-4.

23. Sibiryak SV, Yusupova RS, Kayumova EU. Fas/Apo-1 (CD95) Antigen expression on the peripheral blood lymphocytes in healthy donors and pulmonary tuberculosis patients. Russ J Immunol 1999; 4: 33-42.

24. Zahran WA, Ghonaim MM, Koura BA, El-Banna H, Ali SM, El-Sheikh N. Human natural killer T cells (NKT), NK and T cells in pulmonary tuberculosis: potential indicators for disease activity and prognosis. Egypt J Immunol 2006; 13: 67-78.

25. Behar SM, Porcelli SA. CD1-restricted T cells in host defense to infectious diseases. Curr Top Microbiol Immunol 2007; 314: 215-50.

26. Nishimura T, Kitamura $\mathrm{H}$, Iwakabe $\mathrm{K}$, et al. The interface between innate and acquired immunity: glycolipid antigen presentation by CD1d-expressing dendritic cells to NKT cells induces the differentiation of antigen-specific cytotoxic T lymphocytes. Int Immunol 2000; 12: 987-94.

27. Lockhart E, Green AM, Flynn JL. IL-17 production is dominated by gamma-delta T cells rather than CD4 T cells during Mycobacterium tuberculosis infection. J Immunol 2006; 177: 4662-9.

28. Lee J, Choi K, Olin MR, Cho SN, Molitor TW. Gammadelta $T$ cells in immunity induced by Mycobacterium bovis bacillus Calmette-Guerin vaccination. Infect Immun 2004; 72: 1504-11. 